\title{
Comparative analyses of vertebrate posterior HoxD clusters reveal atypical cluster architecture in the caecilian Typhlonectes natans
}

\author{
An Mannaert ${ }^{1}$, Chris T Amemiya ${ }^{2}$, Franky Bossuyt ${ }^{1 *}$
}

\begin{abstract}
Background: The posterior genes of the HoxD cluster play a crucial role in the patterning of the tetrapod limb. This region is under the control of a global, long-range enhancer that is present in all vertebrates. Variation in limb types, as is the case in amphibians, can probably not only be attributed to variation in Hox genes, but is likely to be the product of differences in gene regulation. With a collection of vertebrate genome sequences available today, we used a comparative genomics approach to study the posterior HoxD cluster of amphibians. A frog and a caecilian were included in the study to compare coding sequences as well as to determine the gain and loss of putative regulatory sequences.

Results: We sequenced the posterior end of the HoxD cluster of a caecilian and performed comparative analyses of this region using HoxD clusters of other vertebrates. We determined the presence of conserved non-coding sequences and traced gains and losses of these footprints during vertebrate evolution, with particular focus on amphibians. We found that the caecilian HoxD cluster is almost three times larger than its mammalian counterpart. This enlargement is accompanied with the loss of one gene and the accumulation of repeats in that area. A similar phenomenon was observed in the coelacanth, where a different gene was lost and expansion of the area where the gene was lost has occurred. At least one phylogenetic footprint present in all vertebrates was lost in amphibians. This conserved region is a known regulatory element and functions as a boundary element in neural tissue to prevent expression of Hoxd genes.

Conclusion: The posterior part of the HoxD cluster of Typhlonectes natans is among the largest known today. The loss of Hoxd-12 and the expansion of the intergenic region may exert an influence on the limb enhancer, by having to bypass a distance seven times that of regular HoxD clusters. Whether or not there is a correlation with the loss of limbs remains to be investigated. These results, together with data on other vertebrates show that the tetrapod Hox clusters are more variable than previously thought.
\end{abstract}

\section{Background}

Perhaps the best studied gene clusters in animals are the Hox clusters, not only for their importance in the establishment of the metazoan body plan, but also for their tight genomic organization. Hox genes encode transcription factors that belong to the family of homeodomain proteins and play an essential role in the establishment of the anterior-posterior body axis during embryonic development. In addition, they are also involved in

\footnotetext{
* Correspondence: fbossuyt@vub.ac.be

'Biology Department, ECOL, Amphibian Evolution Lab, Vrije Universiteit Brussel, Brussels, Belgium

Full list of author information is available at the end of the article
}

patterning of limbs and in organogenesis [1-4]. In vertebrates, the expression domains of Hox genes are collinear in space and time, and reflect their chromosomal arrangement [5].

Invertebrates possess one - often interrupted or disintegrated - Hox cluster, while all vertebrates have multiple clusters [6-8]. Gnathostomes typically have four Hox clusters that arose by subsequent duplications in the stem lineage of vertebrates, while most ray-finned fishes contain seven (e.g. zebrafish) to thirteen (e.g. salmon) clusters as a result of additional, teleost-specific genome duplications and subsequent cluster losses [9-11]. Due to these additional duplications, the gene content of the

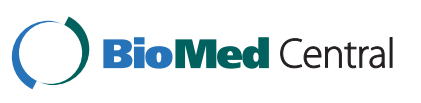

(c) 2010 Mannaert et al; licensee BioMed Central Ltd. This is an Open Access article distributed under the terms of the Creative Commons Attribution License (http://creativecommons.org/licenses/by/2.0), which permits unrestricted use, distribution, and reproduction in any medium, provided the original work is properly cited. 
fish Hox clusters is variable, with different gene losses in different species examined. In contrast, the Hox complement of tetrapods is rather conserved, with the same genes present in mammalian and bird genomes [7]. In the genome of the frog Silurana tropicalis, at least one and possibly two genes have been lost $[12,13]$.

Hox gene clusters in vertebrates are compact (around $100 \mathrm{~kb}$ in mammals and even shorter in teleost fishes), with highly conserved distances between paralogous genes, and with little or no interspersed repetitive DNA elements [14]. The only exceptions known so far are squamate reptiles, with the lizard Anolis carolinensis as a striking example of having accumulated a substantial number of retrotransposons in its Hox clusters, resulting in considerably larger cluster sizes [15,16]. In general, the tight clustering of the Hox genes in vertebrates may be the result of an evolutionary constraint to keep the genes in close proximity, thus maintaining the intergenic distances and prohibiting insertion of interspersed repeats [17]. This constraint may be facilitated by the presence of cis-regulatory elements within the clusters that are shared by neighboring genes, as well as by remote enhancers producing regulatory landscapes that would be broken when the clusters split [18]. In this context, a suite of global long-range enhancers that control the expression of six genes located 5' of the HoxD cluster of fishes and mammals has been discovered [19]. This Global Control Region (GCR) reinforces the effect of another enhancer, Prox, that drives the expression of the genes $L n p$ and $E v x-2$ - both adjacent to the 5 ' end of the HoxD cluster - and the posterior Hoxd genes (Hoxd-13 to Hoxd-10) in the distal limb and genital buds (digit enhancer); it also regulates the expression of $L n p$ and $E v x-2$ in the central nervous system (neural enhancer) [19-21]. The action of the GCR in the nervous system is somehow restricted by boundary elements between $E v x-2$ and Hoxd-13, while in the limb bud its effect decreases progressively with distance from the 5' end of the HoxD cluster [22-24].

Defects in Hoxd genes or gene regulation often have an effect on limb development. For example, the mouse Ulnaless mutation causes reduction of the zeugopod, which is the result of the alteration of Hoxd gene expression due to an inversion of the HoxD cluster and subsequent change of cis-regulatory control $[19,25,26]$. Similar phenotypes can be observed in human mesomelic dysplasia patients, which results from microduplications in the HoxD cluster [27]. Normal limb development can also be affected by mutations within Hoxd genes. For example, the expansion of a poly-alanine tract in HOXD13 results in the synpolydactyly syndrome, with abnormal reductions, duplications and fusions of digits [28].
Despite their differences in limb types, frogs and salamanders are characterized by the presence of only four fingers. Of all amphibians, caecilians (Gymnophiona) probably form the most enigmatic order, as most of them spend their life hidden under the ground. Similar to snakes, they have an elongated trunk and have undergone secondary loss of limbs. The diversity in body plan and limbs among the three amphibian orders may have been affected by changes in Hox gene sequence, or, more likely, regulation (e.g. [29]).

At present, the only amphibian genome sequence publicly available is from the frog Silurana tropicalis. We constructed a BAC library of the aquatic caecilian Typhlonectes natans to obtain the sequence of the posterior end of the HoxD cluster, including Evx-2, i.e. the part of the cluster that is important in limb development and which has been shown to be under control of the Global Control Region. Comparative analyses of this region with the orthologous region of other vertebrates and the subsequent identification of conserved, non-coding, putative regulatory elements may shed light on the evolution of the caecilian body plan.

\section{Results and Discussion}

\section{BAC sequencing and annotation}

The haploid genome size of Typhlonectes natans was estimated at $13.37 \mathrm{pg}$ by flow cytometry with chicken erythrocyte nuclei as an internal standard (data not shown). A pooled BAC library of about 460,000 clones with an average insert size of $107 \mathrm{~kb}$ from Typhlonectes natans was constructed, comprising a theoretical $3.8 \times$ coverage of the genome, and screened by PCR to isolate a clone that contained the posterior HoxD cluster. A single clone with an estimated size of approximately $115 \mathrm{~kb}$ containing Hoxd-13 was sequenced using 454 sequencing technology [30] and over 15,000 reads were assembled into two supercontigs. The orientation of the two supercontigs was determined by sequencing of both BAC ends, and they were assembled into one final contig with a small gap arbitrarily set at 100 base pairs (bp) because the total sequence length is consistent with the estimated insert size. The total caecilian sequence comprises $116,633 \mathrm{bp}$, including a $100 \mathrm{bp}$ gap (GenBank HQ398255). An initial blastx analysis and the software GenomeScan [31] identified the genes Evx-2, Hoxd-13, Hoxd-11 and a large part of Hoxd-10. The exon-intron boundaries were refined manually by alignment with Hox sequences of other vertebrates. Hoxd-12 was not found in these analyses.

\section{Vertebrate posterior HoxD cluster comparison}

We compared the posterior HoxD cluster of the caecilian with the clusters of the horn shark, zebrafish, coelacanth, frog, chicken, anole lizard, opossum, dog, 
mouse and human. In the caecilian, the 5' end of the $H o x D$ cluster, starting from the stop codon of $E v x-2$, situated on the reverse strand, until the stop codon of Hoxd-10 is 107 kilo base pairs (kb), which is over 2.5 times larger than the orthologous region in mammals (approximately $40 \mathrm{~kb}$ ) (Figure 1). Apart from the caecilian, the coelacanth and the lizard sequences are also larger than average, with lengths of $82 \mathrm{~kb}$ and $95 \mathrm{~kb}$, respectively. The expansion of the caecilian HoxD cluster is mainly due to the lengthening of the intergenic region between Hoxd-13 and Hoxd-11, which is over six times larger than in the human. In the coelacanth, the intergenic region between $E v x-2$ and Hoxd-12, where the Hoxd-13 gene was lost, is almost four times longer than the corresponding region in mammals. In the anole lizard, not only the HoxD cluster, but also the other three Hox clusters are significantly longer [15].

Global alignment with the other vertebrates uncovered the remains of Hoxd-12 in the caecilian (Figure 2). The presence of multiple frameshift-producing indels and stop codons implies that it is no longer protein coding and has become a pseudogene ( $\psi H o x-d 12)$. As frogs and caecilians comprise the basal split within amphibians [32], the most parsimonious explanation for the absence of Hoxd-12 from the Silurana tropicalis genome and its pseudogenization in the caecilian is an early loss in amphibian evolution with unequal rates of evolution in T. natans and S. tropicalis, although two independent loss events cannot be excluded. We also discovered a pseudogene ( $\psi$ Hoxd-13) in the coelacanth posterior HoxD sequence. A blastx analysis revealed fragments of both exons that could still be aligned with other vertebrate Hoxd-13 genes.

The length of the coding sequences of Hox genes is similar in both amphibian species, except for Hoxd-13, which is over 100 bp shorter in Typhlonectes. In general, most posterior HoxD coding sequences have comparable

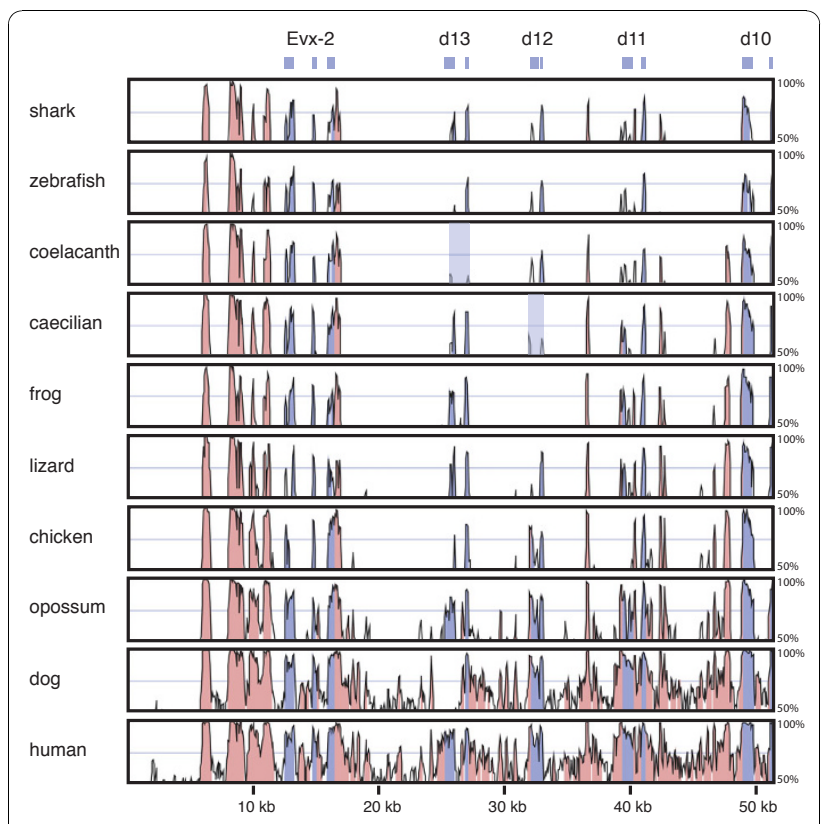

Figure 2 Global alignment of the vertebrate posterior HoxD clusters. The graphs represent the percentage of nucleotide sequence conservation of each vertebrate HoxD cluster compared to the orthologous region in the mouse HoxD cluster. Blue peaks represent coding regions, corresponding to the Hox exons on top of the figure; pink peaks represent highly conserved non-coding sequences. The shaded boxes indicate a Hoxd-13 pseudogene in the coelacanth and a Hoxd-12 pseudogene in the caecilian.

lengths in all species used in this study, except for Hoxd-13 and Hoxd-11, which are about 25\% longer in amniotes and placental mammals, respectively. The amniote or mammalian expansion of the HOXD13 and HOXD11 proteins can be attributed to sequences rich in alanine, glycine, serine and proline. In HOXD13, a homopolymeric repeat consisting of nine alanines in the chicken and 15 alanines in mammals [28] is not present

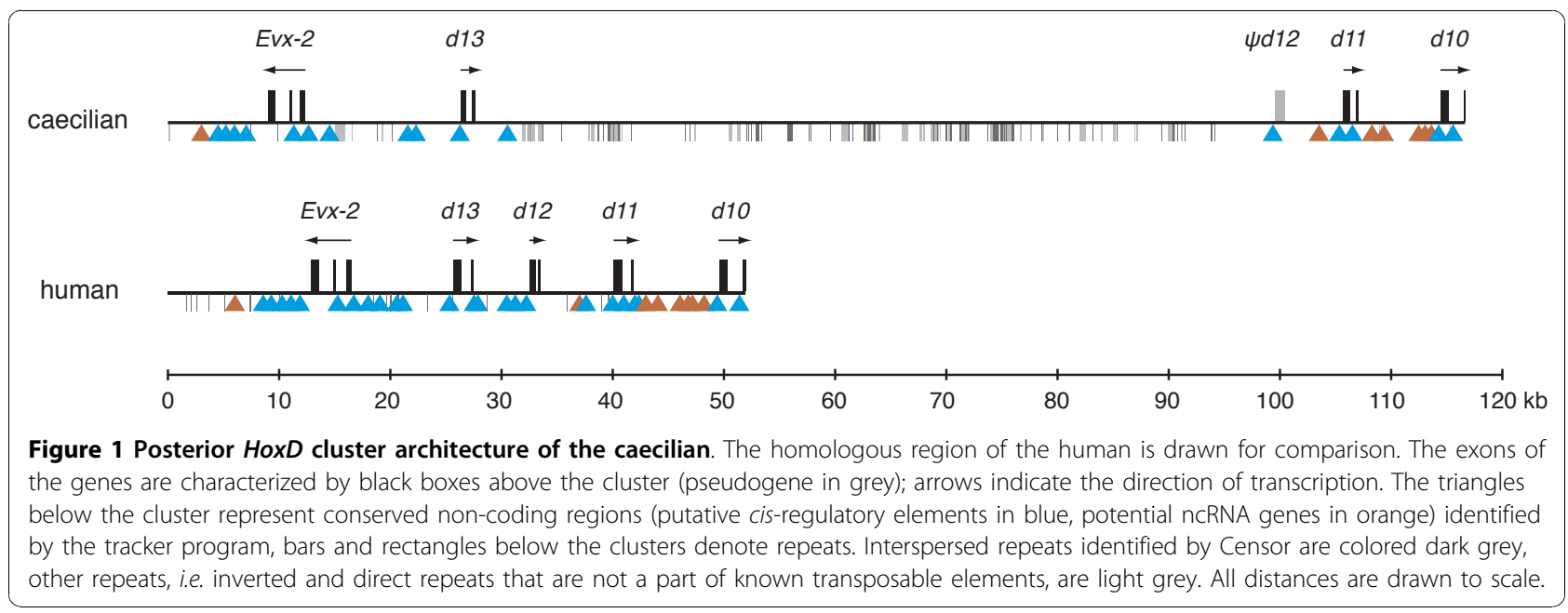


in any other species. HOXD13 proteins are overall rich in serine and alanine in mammals, but not in nonamniotes. In addition to the conservation of both exons of each Hox gene, evolutionarily conserved regions were also detected in non-coding intergenic and intronic sequences. These conserved, non-coding sequences or phylogenetic footprints may represent regulatory elements and non-coding RNA genes and were further investigated.

\section{Distribution of repeats}

To investigate whether the caecilian HoxD cluster expansion was accompanied by an accumulation of repeats, we compared it to the Repbase database [33] of known vertebrate repetitive elements using RepeatMasker [34] and Censor [35]. RepeatMasker identified 4.74\% of the sequence as part of transposable elements, while Censor reports $7.45 \%$ interspersed repeats, mostly retrotransposons like LINES and SINES. It must be noted that the hits are not always very strong because of short alignment length. However, blastn searches of all the repeats resulted in a significant match with known repeats (e-value $\leq 1 \mathrm{e}-05$ ) for 11 out of 54 repeats. Moreover, blastx searches of the intergenic regions recovered one additional SINE and one LINE. The majority of the repeats is located in the intergenic region between Hoxd-13 and $\psi$ Hoxd-12 (Figure 1). The same analyses were performed for the other vertebrate sequences by comparison with the repeat databases for the respective species, if available. In the caecilian and the coelacanth, the repeats are almost exclusively found in the large intergenic region where the pseudogene is situated, i.e. between Hoxd-13 and $\psi$ Hoxd-12 in the caecilian and between $E v x-2$ and $\psi H o x d-13$ in the coelacanth. In the lizard and zebrafish, transposable elements are dispersed over the cluster, with a concentration of repeats between $E v x-2$ and Hoxd-13 in the latter. Additionally, a self-self blastn analysis was performed to identify direct and inverted repeats that are not part of known transposons. This revealed the presence of 30 inverted repeats (stemloop), 15 palindromes (stem) and 12 direct repeats (minimum identity of $70 \%$, e-value $\leq 1 \mathrm{e}-5$ ) in the caecilian $H o x D$ cluster. Again, almost all repeats are located in the region between Hoxd-13 and the HHoxd-12. Some of these repeats are a part of transposable elements, but the majority is unknown. A similar result was obtained for the coelacanth sequence. Here, no direct repeats, 21 inverted repeats and 11 palindromes were found, all located in the region between $E v x-2$ and Hoxd-12, with several large stretches in the former intron of $\psi H o x d-13$. The anole lizard sequence also contains a large amount of inverted and direct repetitive sequences, but spread across the cluster, which is in concordance with the distribution of transposable elements. Few or no additional repeats were found in the other vertebrate $\operatorname{HoxD}$ sequences. The presence of interspersed, repetitive DNA may undermine genomic stability [36]. In vertebrate genomes, regions containing developmental genes, such as Hox clusters, are usually devoid of transposable elements [37], suggesting the presence of a constraint against the invasion of foreign elements into a region essential for development. The caecilian, coelacanth, anole lizard and zebrafish have a higher than average amount of repetitive elements in the posterior $\operatorname{HoxD}$ cluster, and the caecilian and coelacanth also experienced the loss of a functional Hox gene. Whether the presence of repeats attributed to the pseudogenization of Hoxd-12 in the caecilian (and of Hoxd-13 in the coelacanth), or whether the loss of Hoxd-12 weakened the constraint and allowed repeats to accumulate, remains unknown. In the human genome, pseudogenes are frequently found in the vicinity of long inverted repeats [38].

Despite being essential for proper embryonic development, the loss of one Hox gene does not necessarily have a negative impact on an organism's phenotype, as Hox genes can be functionally equivalent [39]. However, expansion of the cluster may have an effect on gene regulation. Since all the genes in this region of the HoxD cluster are under the control of the long-range enhancer GCR, the loss of Hoxd-12 accompanied with the enlargement of the distance between Hoxd-13 and Hoxd-11 and the accumulation of repetitive elements in the caecilian may have an influence on the effect of the GCR on the expression of Hoxd-11 and Hoxd-10. Whether this effect, if any, is reflected in morphology, is unknown, but it is worth mentioning that a similar cluster architecture was found in the corn snake [16]. Some transposable elements have been exapted to modulate gene regulatory networks (reviewed by [40]). As changes in - especially developmental - gene regulation may lead to morphological changes [41], the adoption of a highly derived body plan, such as in caecilians, may have been facilitated by transposable elements. It is possible that caecilians and snakes have employed a similar mechanism of limb loss, though this is probably not the case in other limbless squamates, since Hoxd-12 is present in the slowworm Anguis fragilis [16].

\section{Identification of phylogenetic footprints}

More and more regulatory sequences and non-coding RNA (ncRNA) genes are being discovered in the portion of the genome that does not code for proteins [42]. Although it has been shown that not every regulatory sequence is evolutionarily conserved and that an apparent function cannot always easily be allocated to a conserved sequence $[43,44]$, screening genomes for evolutionarily conserved non-coding sequences is a widely 
used strategy to discover potential regulatory elements. Such elements are expected to be present in the vicinity of transcription factors or developmental genes, such as Hox genes [42]. We identified 33 evolutionarily conserved non-protein coding sequences, or so-called phylogenetic footprints, with the software tracker [45] (Additional file 1). To distinguish putatively transcribed footprints, we blast searched all footprints against the NCBI database of ESTs, which resulted in the identification of eight footprints between 29 and 876 bp long, not located within untranslated regions (UTRs), with one or more EST matches (Additional file 2). Because EST data are absent for many organisms, including coelacanth and caecilians, we did not find ESTs corresponding to these footprints for every organism. However, given the high degree of sequence conservation and the finding of ESTs of each footprint in at least two different organisms, we believe it is possible that transcription and perhaps the function of these elements are conserved. Therefore, we consider these footprints to be putative ncRNA genes, and the other, non-transcribed footprints as potential cis-regulatory elements (Figure 1).

Non-coding RNAs are functional molecules that are not translated into a protein. Instead, they are involved in post-transcriptional modification or DNA replication or have a regulatory function, and can be found in intergenic regions, introns and even in the UTR of genes or overlapping with protein coding genes [46]. The gene regulatory RNAs are usually small and act in trans by post-transcriptional silencing of target genes through the binding of complementary sites. Next to the large number of small ncRNAs, an increasing number of long ncRNAs is being described, which are at least 200 to over 10,000 nucleotides long (reviewed by [47]). Long ncRNAs can act independently of a target sequence, in cis, by interfering with the transcription of a neighboring gene, or in trans, by recruiting proteins that alter the chromatin state [47]. One example of a regulatory ncRNA in the HoxC cluster is HOTAIR, which epigenetically represses transcription of $40 \mathrm{~kb}$ across the HoxD locus [48]. Screening of all the expressed footprints against the Functional RNA Database [49] did not result in the identification of any classified ncRNA. However, four of them produced significant matches with putative RNAs predicted by Evofold, which is a method to identify functional RNA structures in vertebrates by using a combined probabilistic model of RNA structure and sequence evolution [50]. In addition, in footprint fp1, also known as CR3 [51], a significant RNA secondary structure was predicted $(\mathrm{p}=0.92)$. Seven of the expressed footprints lie in the intergenic region between Hoxd-11 and Hoxd-10 (Figure 1). What tracker considers to be multiple footprints may correspond to a single potential ncRNA gene, as indicated by alignment of the region between Hoxd-11 and Hoxd-10 with the corresponding ESTs (not shown). Two of the expressed footprints were previously identified as regulatory regions RVIII/RIX (fp26) and RX (fp20) ([52-55]. The fact that these two regulatory regions appear to be transcribed may shed new light on how they function.

Cis-regulatory elements can be anticipated in intergenic regions, in introns and in the 5' and 3' UTR of protein-coding genes, and may act as a promoter, enhancer, repressor or insulator [42]. In total, 24 footprints (fps) are putative cis-regulatory elements. Nine of these were found in the intergenic regions, including the area downstream of $E v x-2$; four footprints are located in an intron, five located in the 5' UTR and six located in the 3' UTR of the Hox and Evx-2 genes. Three of the intergenic footprints were identified in previous studies and are known as RXI (fp18) and RXII (fp9 and fp10) $[23,56]$. Two other intergenic footprints (fp 2 and fp3) are extremely conserved in all species used in this study and are located downstream $E v x-2$. The reason for this high degree of conservation however remains unclear [51]. One footprint (fp23) that was found in the intron of Hoxd-11 contains a HB1 element, which consists of homeodomain binding sites. This element is previously described from the intron of Hoxa7, the Drosophila homolog $U b x$, the introns of Hox4 genes and the intron of Hoxa-11 [57-60]. Of the five footprints in the 5'UTR, two also include the promoter region. The 5' and 3' UTRs of mRNAs play an important role in the posttranscriptional regulation of gene expression through the presence of cis-acting elements and through interaction with micro-RNAs (miRNAs) $[61,62]$. Conserved sequences in 3' UTRs may contain potential target sites for miRNAs that are involved in post-transcriptional gene silencing. Micro-RNAs are short, single-stranded RNA molecules of $\sim 22$ nucleotides that show at least partial complementarities to their target mRNA. If the miRNA is only partially complementary, a perfect match between the seed (nucleotides 2 to 7 ) of the miRNA and the target mRNA is necessary for inhibition of translation or for promotion of deadenylation $[63,64]$. Therefore all six footprints located in the 3'UTR of the genes were screened for the presence of hypothetical target sites for miRNAs. One footprint (fp15), located in the 3'UTR of Hoxd-13, contains a short motif of seven nucleotides, with perfect match to the seed of the miRNA miR-26.

\section{Reconstruction of conserved element evolution}

Gains and losses of putative ncRNAs and cis-regulatory elements in vertebrate posterior $H o x D$ clusters were mapped on a vertebrate timescale under the Dollo parsimony criterion, i.e. assuming a single origin (Figure 3). Two putative $c i s$-regulatory elements have been lost in 


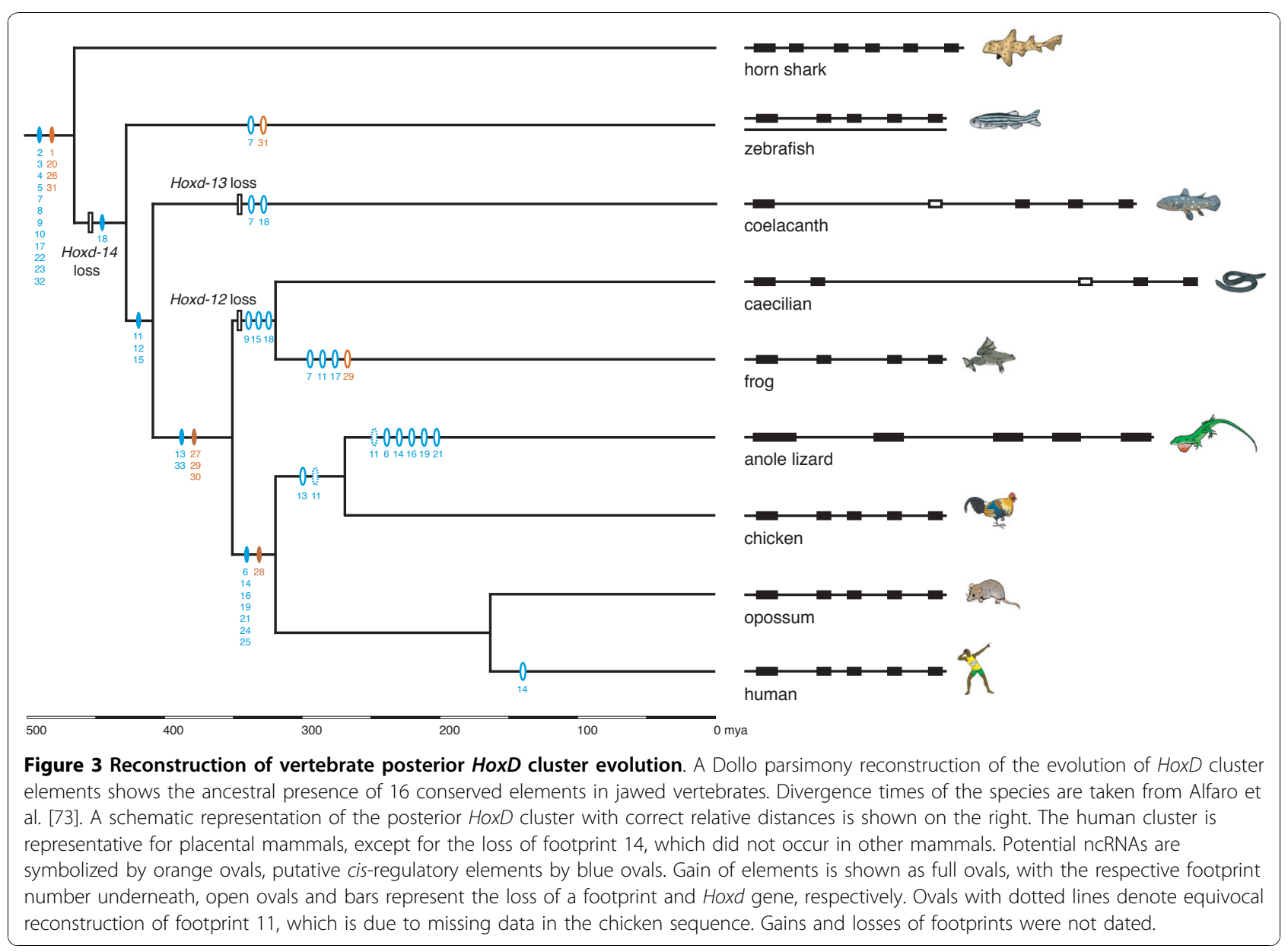

the amphibian ancestor: fp9, which is one of two motifs of RXII, and fp15, a small footprint in the 3' UTR of Hoxd-13. The conserved region RXII is a boundary element located in the intergenic region between $E v x-2$ and Hoxd-13, whose promoters are in each other's vicinity. Unlike Hoxd genes, Evx-2 is expressed in the central nervous system, regulated by the neural enhancer of the GCR. RXII is considered to function as an insulator to prevent the ectopic expression of Hoxd genes in the nervous system, since GCR regulation is not promoterspecific [23]. Although tracker failed to recover the entire RXII element (fp9 and fp10) in the zebrafish, short, apparently homologous sequences were found in the zebrafish cluster in the same area as in other vertebrates [23]. Moreover, both footprints were found in the horn shark, which points to their ancestral presence in vertebrates, and therefore we conclude that fp9 was lost in amphibians. Footprint 15 is a short DNA stretch of $\sim 22 \mathrm{bp}$ with a highly conserved motif of $7 \mathrm{bp}$ located in the 3' UTR of Hoxd-13. This motif is somewhat degenerated in the coelacanth, which does not have a functional Hoxd-13 gene, and it is located around $14 \mathrm{~kb}$ 5 ' to $\psi$ Hoxd-13, which may indicate that this sequence is not homologous to the other vertebrate fp15 sequences. On the other hand, pseudogenization of Hoxd-13 and insertion of repetitive sequences may have promoted the relative relocation of this element. For our reconstruction, we considered all footprints as identified by the tracker software to be truly conserved sequences. Therefore, we conclude that this element originated in Sarcopterygii, and was lost in amphibians. In theory, this footprint can serve as a target for the microRNA miR26 , which is expressed in neurons and astrocytes of the developing mouse brain [65]. The absence of both elements may indicate that restriction of Hoxd-13 expression in neural tissue is regulated differently in amphibians.

\section{Conclusion}

The posterior HoxD cluster of the caecilian is much larger than that of most known vertebrates due to the accumulation of interspersed and inverted repeats accompanied with Hoxd-12 gene loss. A similar event occurred in the coelacanth and in the corn snake HoxD cluster. Whether these occurrences are reflected in the morphology of these species is not clear, but it is 
possible that caecilians and snakes adopted a similar mechanism that resulted in body elongation and limb loss. The distance created between two subsequent genes that are under the control of the same long-range limb enhancer is likely to have some effect on the action of this enhancer on the genes after the void. A number of conserved, non-coding regions have been identified in the HoxD cluster, some of them showing extremely high conservation among all vertebrates. While no new footprints arose in the amphibian lineage, at least one, and possibly two conserved sequences were lost. These results, together with data on other vertebrate Hox clusters show that tetrapod Hox clusters show more variation than expected previously.

\section{Methods}

\section{Genomic library and DNA sequencing}

High molecular weight DNA was extracted from erythrocytes from two Typhlonectes natans specimens and was used to construct a BAC library according to Osoegawa et al. [66] and as described by Danke et al. [67]. The genome size was estimated by flow cytometry analysis using chicken erythrocyte nuclei as a standard. EcoRI partial digests of the caecilian DNA were size selected and inserted in the $\mathrm{PCC} 1 \mathrm{BAC}$ vector (Epicentre) and the library was combined in 2304 pools containing 200 clones each. The library was screened by PCR with primers specific for Hoxd-13 (forward primer: 5'-GCAATGAAGGCGCCTCCAG-3', reverse primer: 5'GGAGATATAGGTGTCGTGCCTCGG-3') to isolate the posterior end of the HoxD cluster. The positive clone was 454 GS FLX-sequenced and assembled by Eurofins MWG Operon (Ebersberg, Germany). The clone was also end sequenced using BigDye 3.1 chemistry on an ABI 3100 Genetic Analyzer. Several smaller contigs were assembled manually and contig overlaps were confirmed by PCR, except in one case where a gap was present. Because the length of the two contigs is consistent with the size of the insert, we inferred this gap to be small and therefore arbitrarily set it at $100 \mathrm{bp}$.

\section{Caecilian HoxD cluster annotation}

The genes in the caecilian posterior HoxD cluster were annotated by initial blastx searches of the entire cluster sequence and with the program GenomeScan [31] using mouse HOXD and EVX2 proteins as a training set. Exon - intron boundaries were determined manually by alignment with Hox sequences of other vertebrates.

\section{HoxD alignments}

Global alignments of the caecilian posterior HoxD cluster with other vertebrate $\operatorname{HoxD}$ clusters were performed with MultiPipMaker [68] and VISTA [69]. The HoxD clusters of the following species were used: Heterodontus francisci (horn shark, AF224263), Danio rerio (zebrafish, UCSC Genome Browser) Latimeria menadoensis (Indonesian coelacanth, FJ497008), Silurana tropicalis (tropical clawed frog, JGI), Gallus gallus (chicken, ENSEMBL), Anolis carolinensis (green anole, UCSC Genome Browser), Monodelphis domestica (grey shorttailed opossum, ENSEMBL), Canis familiaris (domestic dog, ENSEMBL), Homo sapiens (human, NT_005403) and Mus musculus (mouse, AC_015584). The MultiPipMaker alignments were performed with mouse and $T$. natans as reference sequence respectively.

Interspersed repeats and low complexity regions in all clusters were masked by screening against a library of repetitive elements if available for the organism by RepeatMasker [34]. If no such library was available, as for the horn shark, coelacanth, caecilian, lizard and opossum, the sequences were compared to a database of transposable element encoded proteins.

\section{Repeat content}

The repeat content of the HoxD clusters was determined with Censor [35] and RepeatMasker, using the Repbase library of the species-specific or vertebrate repeats and a database of transposable element encoded proteins. All reported interspersed repeats were taken into account. In addition, self-self blastn analyses were performed to identify direct and inverted repeats within each cluster. Only repeats with maximum $30 \%$ mismatch and an e-value $\leq 1 \mathrm{e}-05$ were retained.

\section{Analyses of phylogenetic footprints}

The program tracker [45] was used to detect evolutionarily conserved non-coding sequences or phylogenetic footprints. This program is based on blastz [70] to produce initial local pairwise alignments of all pairs of the input sequences. Only the intergenic regions between two homologous genes are compared. After several filtering steps, these alignments, which contain a window of 12 nucleotides with minimum identity of $75 \%$, are assembled into groups of partially overlapping regions, resulting in local sequence alignments, or footprint cliques. To be able to detect conserved sequences in all non-coding regions, introns were treated as intergenic regions. This analysis was repeated using the same, but repeat masked sequences and all footprints from both analyses were combined. Not every footprint is necessarily conserved in each taxon, as its presence or absence may be indicative of the loss of a cis-regulatory element and a subsequent change in gene regulation. Phylogenetic footprints were treated as two classes: potential (cis-) regulatory elements and putative non-coding RNA genes. Conserved sequences found in the 3' UTR of genes were screened for the presence of hypothetical target sites of miRNAs using TargetScan Release 5.1 
[63,71]. To detect whether footprints are expressed and thus may be ncRNA genes, a blastn search of all footprints against the NCBI database of ESTs was performed. Footprints with one or more EST matches were screened against the Functional RNA Database [49] for similarity with known ncRNAs. In addition, the RNAz server (Vienna RNA server, http://rna.tbi.univie.ac.at) was used to identify thermodynamically stable and evolutionarily conserved RNA secondary structures in the footprint alignments.

A Dollo parsimony reconstruction of the genes and footprints was done with MacClade v4.06 [72] to assess whether putative cis-regulatory elements and ncRNAs were lost or gained during vertebrate evolution.

\section{Additional material}

\section{Additional file 1: Summary of the conserved sequences in} vertebrate Hox clusters. This table gives an overview of the positions of the coding regions in the vertebrate sequences as well as all footprint positions identified by the tracker software. The footprints are named $\mathrm{fp} 1$ to fp33, and footprints that are expressed in at least two species are indicated in bold.

Additional file 2: EST blast hits of expressed footprints. All ESTs that correspond to the expressed footprints are given with Genbank accession number, species name and tissue source.

\section{Acknowledgements}

We thank Joel Sohn for help with the caecilians, Andrew Stuart and the other members of the Amemiya Lab for technical assistance and Aru for genome size measurements. We are grateful to Kim Roelants for the drawings and to Wolfgang Otto for help with the tracker software. AM was supported by a Travel Grant of the Research Foundation - Flanders (FWO) for her stay in Seattle. FB and AM received financial support from the Vrije Universiteit Brussel (Concerted Research Action 29).

\section{Author details}

'Biology Department, ECOL, Amphibian Evolution Lab, Vrije Universiteit Brussel, Brussels, Belgium. 'Benaroya Research Institute at Virginia Mason and University of Washington, Seattle, USA.

\section{Authors' contributions}

The research was designed by AM and FB. AM conducted the laboratory work, except the 454 sequencing; CTA provided assistance with the BAC library construction. AM performed the analyses. All authors wrote the manuscript.

Received: 11 June 2010 Accepted: 24 November 2010

Published: 24 November 2010

\section{References}

1. McGinnis W, Krumlauf R: Homeobox genes and axial patterning. Cell 1992, 68(2):283-302

2. Zakany J, Duboule D: The role of Hox genes during vertebrate limb development. Curr Opin Genet Dev 2007, 17(4):359-366.

3. Kondo T, Dollé P, Zákány J, Duboule D: Function of posterior HoxD genes in the morphogenesis of the anal sphincter. Development 1996, 122:2651-2659.

4. Di-Poï N, Zákány J, Duboule D: Distinct roles and regulations for Hoxd genes in metanephric kidney development. PLOS Genet 2007, 3(12):e232.

5. Kmita M, Duboule D: Organizing axes in time and space; 25 years of colinear tinkering. Science 2003, 301(5631):331-333.
6. Lemons D, McGinnis W: Genomic evolution of Hox gene clusters. Science 2006, 313(5795):1918-1922.

7. Powers TP, Amemiya CT: Evolutionary plasticity of vertebrate Hox genes. Current Genomics 2004, 5(6):459-472.

8. Seo HC, Edvardsen RB, Maeland AD, Bjordal M, Jensen MF, Hansen A, Flaat M, Weissenbach J, Lehrach H, Wincker $P$, et al: Hox cluster disintegration with persistent anteroposterior order of expression in Oikopleura dioica. Nature 2004, 431(7004):67-71.

9. Bailey WJ, Kim J, Wagner GP, Ruddle FH: Phylogenetic reconstruction of vertebrate Hox cluster duplications. Mol Biol Evol 1997, 14(8):843-853.

10. Meyer A, Van de Peer $Y$ : From $2 R$ to $3 R$ : evidence for a fish-specific genome duplication (FSGD). Bioessays 2005, 27(9):937-945.

11. Mungpakdee $S$, Seo HC, Angotzi AR, Dong $X$, Akalin A, Chourrout D: Differential evolution of the 13 Atlantic salmon Hox clusters. Mol Biol Evol 2008, 25(7):1333-1343.

12. Hoegg S, Meyer A: Hox clusters as models for vertebrate genome evolution. Trends Genet 2005, 21(8):421-424.

13. Mannaert A, Roelants $K$, Bossuyt F, Leyns L: A PCR survey for posterior Hox genes in amphibians. Mol Phylogenet Evol 2006, 38(2):449-458.

14. Fried C, Prohaska SJ, Stadler PF: Exclusion of repetitive DNA elements from gnathostome Hox clusters. J Exp Zoolog B Mol Dev Evol 2004, 302(2):165-173.

15. Di-Poï N, Montoya-Burgos Jl, Duboule D: Atypical relaxation of structural constraints in Hox gene clusters of the green anole lizard. Genome Res 2009, 19(4):602-610.

16. Di-Poï N, Montoya-Burgos II, Miller H, Pourquié O, Milinkovitch MC, Duboule D: Changes in Hox genes' structure and function during the evolution of the squamate body plan. Nature 2010, 464(7285):99-103.

17. Duboule D: The rise and fall of Hox gene clusters. Development 2007, 134(14):2549-2560

18. van der Hoeven F, Zakany J, Duboule D: Gene transpositions in the HoxD complex reveal a hierarchy of regulatory controls. Cell 1996, 85(7):1025-1035

19. Spitz F, Gonzalez F, Duboule D: A global control region defines a chromosomal regulatory landscape containing the HoxD cluster. Cell 2003, 113(3):405-417.

20. Gonzalez F, Duboule D, Spitz F: Transgenic analysis of Hoxd gene regulation during digit development. Dev Biol 2007, 306(2):847-859.

21. Spitz F, Duboule D: Global control regions and regulatory landscapes in vertebrate development and evolution. Adv Genet 2008, 61:175-205.

22. Kmita M, Fraudeau N, Herault Y, Duboule D: Serial deletions and duplications suggest a mechanism for the collinearity of Hoxd genes in limbs. Nature 2002, 420(6912):145-150.

23. Kmita M, Tarchini B, Duboule D, Herault Y: Evolutionary conserved sequences are required for the insulation of the vertebrate Hoxd complex in neural cells. Development 2002, 129(23):5521-5528.

24. Monge I, Kondo T, Duboule D: An enhancer-titration effect induces digitspecific regulatory alleles of the HoxD cluster. Dev Biol 2003, 256(2):212-220.

25. Herault $Y$, Fraudeau N, Zakany J, Duboule D: Ulnaless (UI), a regulatory mutation inducing both loss-of-function and gain-of-function of posterior Hoxd genes. Development 1997, 124(18):3493-3500.

26. Peichel $\mathrm{CL}$, Prabhakaran $\mathrm{B}$, Vogt TF: The mouse Ulnaless mutation deregulates posterior HoxD gene expression and alters appendicular patterning. Development 1997, 124(18):3481-3492.

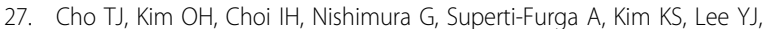
Park WY: A dominant mesomelic dysplasia associated with a $1.0-\mathrm{Mb}$ microduplication of HOXD gene cluster at 2q31.1. J Med Genet 2010, 47(9):638-639

28. Muragaki Y, Mundlos S, Upton J, Olsen BR: Altered growth and branching patterns in synpolydactyly caused by mutations in HOXD13. Science 1996, 272(5261):548-551

29. Vinagre T, Moncaut N, Carapuco M, Novoa A, Bom J, Mallo M: Evidence for a myotomal Hox/Myf cascade governing nonautonomous control of rib specification within global vertebral domains. Dev Cell 2010, 18(4):655-661.

30. Margulies M, Egholm M, Altman WE, Attiya S, Bader JS, Bemben LA, Berka J, Braverman MS, Chen YJ, Chen Z, et al: Genome sequencing in microfabricated high-density picolitre reactors. Nature 2005, 437(7057):376-380. 
31. Yeh RF, Lim LP, Burge CB: Computational inference of homologous gene structures in the human genome. Genome Res 2001, 11(5):803-816.

32. Zardoya $R$, Meyer A: On the origin of and phylogenetic relationships among living amphibians. Proc Natl Acad Sci USA 2001, 98(13):7380-7383.

33. Jurka J, Kapitonov W, Pavlicek A, Klonowski P, Kohany O, Walichiewicz J: Repbase Update, a database of eukaryotic repetitive elements. Cytogenet Genome Res 2005, 110(1-4):462-467.

34. Smit AFA, Hubley R, Green P: Repeatmasker Open-3.0. 1996 [http://www. repeatmasker.org].

35. Kohany O, Gentles AJ, Hankus L, Jurka J: Annotation, submission and screening of repetitive elements in Repbase: RepbaseSubmitter and Censor. BMC Bioinformatics 2006, 7:474.

36. Gordenin DA, Lobachev KS, Degtyareva NP, Malkova AL, Perkins E, Resnick MA: Inverted DNA repeats: a source of eukaryotic genomic instability. Molecular and Cellular Biology 1993, 13(9):5315-5322.

37. Simons C, Makunin IV, Pheasant M, Mattick JS: Maintenance of transposonfree regions throughout vertebrate evolution. BMC Genomics 2007, 8:470.

38. Wang $Y$, Leung FCC: A study on genomic distribution and sequence features of human long inverted repeats reveals species-specific intronic inverted repeats. FEBS Journal 2009, 276(7):1986-1998.

39. Wellik DM, Capecchi MR: Hox10 and Hox11 genes are required to globally pattern the mammalian skeleton. Science 2003 301(5631):363-367.

40. Bourque $\mathrm{G}$ : Transposable elements in gene regulation and in the evolution of vertebrate genomes. Curr Opin Genet Dev 2009, 19(6):607-612.

41. Carroll SB: Chance and necessity: the evolution of morphological complexity and diversity. Nature 2001, 409(6823):1102-1109.

42. Elgar $G$, Vavouri $T$ : Tuning in to the signals: noncoding sequence conservation in vertebrate genomes. Trends Genet 2008, 24(7):344-352.

43. Ahituv N, Zhu Y, Visel A, Holt A, Afzal V, Pennacchio LA, Rubin EM: Deletion of ultraconserved elements yields viable mice. PLoS Biol 2007, 5(9):e234

44. McGaughey DM, Vinton RM, Huynh J, Al-Saif A, Beer MA, McCallion AS: Metrics of sequence constraint overlook regulatory sequences in an exhaustive analysis at phox2b. Genome Res 2008, 18(2):252-260.

45. Prohaska SJ, Fried C, Flamm C, Wagner GP, Stadler PF: Surveying phylogenetic footprints in large gene clusters: applications to Hox cluster duplications. Mol Phylogenet Evol 2004, 31(2):581-604.

46. Mattick JS: The genetic signatures of noncoding RNAs. PLOS Genet 2009 5(4):e1000459.

47. Mercer TR, Dinger ME, Mattick JS: Long non-coding RNAs: insights into functions. Nat Rev Genet 2009, 10(3):155-159.

48. Rinn JL, Kertesz M, Wang JK, Squazzo SL, Xu X, Brugmann SA, Goodnough LH, Helms JA, Farnham PJ, Segal E, et al: Functional demarcation of active and silent chromatin domains in human HOX loci by noncoding RNAs. Cell 2007, 129(7):1311-1323.

49. Mituyama $T$, Yamada $K$, Hattori E, Okida H, Ono $Y$, Terai G, Yoshizawa A, Komori T, Asai K: The Functional RNA Database 3.0: databases to support mining and annotation of functional RNAs. Nucleic Acids Res 2009, , 37 Database: D89-92.

50. Pedersen JS, Bejerano G, Siepel A, Rosenbloom K, Lindblad-Toh K, Lander ES, Kent J, Miller W, Haussler D: Identification and classification of conserved RNA secondary structures in the human genome. PLOS Comput Biol 2006, 2(4):e33.

51. Sabarinadh C, Subramanian S, Tripathi A, Mishra RK: Extreme conservation of noncoding DNA near HoxD complex of vertebrates. BMC Genomics 2004, 5(1):75.

52. Gérard M, Duboule D, Zakany J: Structure and activity of regulatory elements involved in the activation of the Hoxd-11 gene during late gastrulation. Embo J 1993, 12(9):3539-3550.

53. Gérard M, Chen JY, Gronemeyer H, Chambon P, Duboule D, Zakany J: In vivo targeted mutagenesis of a regulatory element required for positioning the Hoxd-11 and Hoxd-10 expression boundaries. Genes Dev 1996, 10(18):2326-2334.

54. Beckers J, Gerard M, Duboule D: Transgenic analysis of a potential Hoxd$11 \mathrm{limb}$ regulatory element present in tetrapods and fish. Dev Biol 1996, 180(2):543-553.

55. Beckers J, Duboule D: Genetic analysis of a conserved sequence in the HoxD complex: regulatory redundancy or limitations of the transgenic approach? Dev Dyn 1998, 213(1):1-11.
56. Hérault $Y$, Beckers J, Kondo T, Fraudeau N, Duboule D: Genetic analysis of a Hoxd-12 regulatory element reveals global versus local modes of controls in the HoxD complex. Development 1998, 125(9):1669-1677.

57. Morrison A, Chaudhuri C, Ariza-McNaughton L, Muchamore I, Kuroiwa A, Krumlauf R: Comparative analysis of chicken Hoxb-4 regulation in transgenic mice. Mech Dev 1995, 53(1):47-59.

58. Haerry TE, Gehring WJ: Intron of the mouse Hoxa-7 gene contains conserved homeodomain binding sites that can function as an enhancer element in Drosophila. Proc Natl Acad Sci USA 1996, 93(24):13884-13889.

59. Haerry TE, Gehring WJ: A conserved cluster of homeodomain binding sites in the mouse Hoxa-4 intron functions in Drosophila embryos as an enhancer that is directly regulated by Ultrabithorax. Dev Biol 1997, 186(1):1-15.

60. Santini $\mathrm{S}$, Boore $J \mathrm{~L}$, Meyer A: Evolutionary conservation of regulatory elements in vertebrate Hox gene clusters. Genome Res 2003, 13(6A):1111-1122.

61. Mignone F, Gissi C, Liuni S, Pesole G: Untranslated regions of mRNAs. Genome Biol 2002, 3(3):REVIEWS0004.

62. Flynt AS, Lai EC: Biological principles of microRNA-mediated regulation: shared themes amid diversity. Nat Rev Genet 2008, 9(11):831-842.

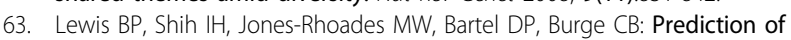
mammalian microRNA targets. Cell 2003, 115(7):787-798.

64. Bartel DP: MicroRNAs: genomics, biogenesis, mechanism, and function. Cell 2004, 116(2):281-297.

65. Smirnova L, Grafe A, Seiler A, Schumacher S, Nitsch R, Wulczyn FG: Regulation of miRNA expression during neural cell specification. Eur Neurosci 2005, 21(6):1469-1477.

66. Osoegawa K, Woon PY, Zhao B, Frengen E, Tateno M, Catanese JJ, de Jong PJ: An improved approach for construction of bacterial artificial chromosome libraries. Genomics 1998, 52(1):1-8.

67. Danke J, Miyake T, Powers T, Schein J, Shin H, Bosdet I, Erdmann M, Caldwell R, Amemiya $C T$ : Genome resource for the Indonesian coelacanth, Latimeria menadoensis. J Exp Zoolog A Comp Exp Biol 2004, 301(3):228-234.

68. Schwartz S, Elnitski L, Li M, Weirauch M, Riemer C, Smit A, Green ED, Hardison RC, Miller W: MultiPipMaker and supporting tools: Alignments and analysis of multiple genomic DNA sequences. Nucleic Acids Res 2003, 31(13):3518-3524.

69. Mayor C, Brudno M, Schwartz JR, Poliakov A, Rubin EM, Frazer KA Pachter LS, Dubchak I: VISTA: visualizing global DNA sequence alignments of arbitrary length. Bioinformatics 2000, 16(11):1046-1047.

70. Schwartz S, Zhang Z, Frazer KA, Smit A, Riemer C, Bouck J, Gibbs R, Hardison R, Miller W: PipMaker-a web server for aligning two genomic DNA sequences. Genome Res 2000, 10(4):577-586.

71. Friedman RC, Farh KK, Burge CB, Bartel DP: Most mammalian mRNAs are conserved targets of microRNAs. Genome Res 2009, 19(1):92-105.

72. Maddison WP, Maddison DR: MacClade, analysis of phylogeny and character evolution, Version 4.02000

73. Alfaro ME, Santini F, Brock C, Alamillo H, Dornburg A, Rabosky DL, Carnevale G, Harmon LJ: Nine exceptional radiations plus high turnover explain species diversity in jawed vertebrates. Proc Natl Acad Sci USA 2009, 106(32):13410-13414

doi:10.1186/1471-2164-11-658

Cite this article as: Mannaert et al: Comparative analyses of vertebrate posterior HoxD clusters reveal atypical cluster architecture in the caecilian Typhlonectes natans. BMC Genomics 2010 11:658. 